\title{
Tudor staphylococcal nuclease is a structure-specific ribonuclease that degrades RNA at unstructured regions during microRNA decay
}

\author{
CHIA-LUNG LI, ${ }^{1,3}$ WEI-ZEN YANG ${ }^{1,3}$ ZHONGHAO SHI, ${ }^{1}$ and HANNA S. YUAN ${ }^{1,2}$ \\ ${ }^{1}$ Institute of Molecular Biology, Academia Sinica, Taipei, Taiwan 11529, ROC \\ ${ }^{2}$ Graduate Institute of Biochemistry and Molecular Biology, National Taiwan University, Taipei, Taiwan 10048, ROC
}

\begin{abstract}
Tudor staphylococcal nuclease (TSN) is an evolutionarily conserved ribonuclease in eukaryotes that is composed of five staphylococcal nuclease-like domains (SN1-SN5) and a Tudor domain. TSN degrades hyper-edited double-stranded RNA, including primary miRNA precursors containing multiple $I \bullet U$ and $U \bullet I$ pairs, and mature miRNA during miRNA decay. However, how TSN binds and degrades its RNA substrates remains unclear. Here, we show that the C. elegans TSN (cTSN) is a monomeric $\mathrm{Ca}^{2+}$-dependent ribonuclease, cleaving RNA chains at the $5^{\prime}$-side of the phosphodiester linkage to produce degraded fragments with $5^{\prime}$-hydroxyl and $3^{\prime}$-phosphate ends. cTSN degrades single-stranded RNA and double-stranded RNA containing mismatched base pairs, but is not restricted to those containing multiple $I \bullet U$ and $U \bullet I$ pairs. cTSN has at least two catalytic active sites located in the SN1 and SN3 domains, since mutations of the putative $\mathrm{Ca}^{2+}$-binding residues in these two domains strongly impaired its ribonuclease activity. We further show by small-angle X-ray scattering that rice osTSN has a flexible two-lobed structure with open to closed conformations, indicating that TSN may change its conformation upon RNA binding. We conclude that TSN is a structure-specific ribonuclease targeting not only single-stranded RNA, but also unstructured regions of double-stranded RNA. This study provides the molecular basis for how TSN cooperates with RNA editing to eliminate duplex RNA in cell defense, and how TSN selects and degrades RNA during microRNA decay.
\end{abstract}

Keywords: ribonuclease; microRNA decay; RNA editing; RNA silencing; TSN

\section{INTRODUCTION}

Tudor staphylococcal nuclease (TSN; also known as Tudor$\mathrm{SN}, \mathrm{SND1}$, or p100) is a conserved multifunctional protein in eukaryotes that participates in various cellular events, from transcriptional to post-transcriptional regulation. TSN was first identified as a transcription coactivator interacting with transcription factors such as EBNA-2, cMyb, STAT6, and STAT5 to promote cell proliferation and differentiation in mammals (Leverson et al. 1998; Abe et al. 2005; Välineva et al. 2005; Paukku et al. 2007). Subsequently, a positive correlation between carcinogenesis and TSN overexpression was discovered in different types of cancers, including colon cancer (Tsuchiya et al. 2007), hepatocellular carcinoma (Yoo et al. 2011), breast cancer (Blanco et al. 2011; Wan et al. 2014), prostate cancer (Kuruma et al. 2009), and malignant glioma (Emdad et al. 2015). Moreover, TSN is cleaved by caspases and facilitates programmed cell death during development and stress responses (Sundström et al.

\footnotetext{
${ }^{3}$ These authors contributed equally to this work.

Corresponding author: hanna@sinica.edu.tw

Article is online at http://www.rnajournal.org/cgi/doi/10.1261/rna. 064501.117. Freely available online through the RNA Open Access option.
}

2009). TSN also interacts with small ribonucleoproteins and Sm proteins involved in pre-mRNA splicing (Yang et al. 2007; Gao et al. 2012; Cappellari et al. 2014). In plants, TSN is an RNA-binding protein involved in RNA transport and localization, which is essential for stress tolerance and has been linked to the formation of stress granules and processing bodies (Wang et al. 2008; Frei dit Frey et al. 2010; Gutierrez-Beltran et al. 2015). Hence, TSN functions as a scaffold for protein-protein or protein-RNA interactions in transcription regulation, mRNA localization, and premRNA splicing (Gutierrez-Beltran et al. 2016).

Besides functioning as a scaffold protein, TSN also possesses ribonuclease activity linked to microRNA (miRNA) decay. TSN was first identified as a component of the RNA-induced silencing complex (RISC) in Caenorhabditis elegans, Drosophila, and mammals (Caudy et al. 2003); although Argonaute 2 , and not TSN, is the "slicer" in RISC responsible for siRNAguided mRNA degradation during RNA silencing. This finding prompted the question as to which types of RNA are

C $2018 \mathrm{Li}$ et al. This article, published in RNA, is available under a Creative Commons License (Attribution 4.0 International), as described at http:// creativecommons.org/licenses/by/4.0/. 
cleaved by TSN in vivo. A series of interesting studies have shown that X. laevis and human TSN degrades hyper-edited double-stranded RNA (dsRNA) containing multiple $\mathrm{I} \cdot \mathrm{U}$ and U.I pairs generated by ADAR (adenosine deaminase acting on RNA), which converts adenosines to inosines in dsRNA (Scadden and Smith 2001; Scadden 2005). TSN thus likely cooperates with RNA editing to dispose of hyper-edited viral RNA in cell defense. Moreover, some primary miRNA precursors, including the precursor of pri-miR142, are edited by ADAR and these edited RNA are further degraded by TSN in mice, suggesting that TSN plays a role in miRNA decay (Yang et al. 2006). Recently, TSN was further identified to be a ribonuclease that degrades both protein-free and Argonaute 2-loaded mature miRNA that promotes the G1/S phase transition in human cells (Elbarbary et al. 2017b). TSN, therefore, plays an important role in miRNA decay in mammals, targeting not only hyper-edited miRNA precursors, but also mature miRNA.

TSN is composed of four tandem repeats of staphylococcal nuclease-like domains (SN1-4), followed by an SN5 domain incorporating an inserted Tudor domain (Fig. 1A; Callebaut and Mornon 1997). The four tandem SN1-4 domains are responsible for RNA binding and cleavage ( $\mathrm{Li}$ et al. 2008), whereas the Tudor domain interacts with symmetrically dimethylated arginines of small nuclear ribonuceloprotein (snRNP) or Piwi family proteins through its conserved aromatic cage (Shaw et al. 2007; Friberg et al. 2009; Liu et al. 2010; Musiyenko et al. 2012). A crystal structure of the C terminal of human TSN (hTSN) that included four domains (SN3-SN4-Tudor-SN5) revealed a basic concave surface in the SN3-SN4 region that is likely involved in RNA binding (Li et al. 2008). A crystal structure of the N-terminal SN1SN2 domain suggests a unique groove between the SN1 and SN2 domains that binds to the partner protein, Metadherin, and has been linked to the development of breast cancer (Guo et al. 2014). Thus, TSN harbors multiple domains that allow it to play various roles in RNA and protein interactions.

Compared to its bridging roles in protein-protein interactions, how TSN selects its RNA substrates for cleavage is less clear. TSN specifically degrades duplex RNA with $\mathrm{I} \cdot \mathrm{U}$ and $\mathrm{U} \cdot \mathrm{I}$ pairs, which are particularly unstable since duplex RNA containing single or double $\mathrm{I} \cdot \mathrm{U} / \mathrm{U} \cdot \mathrm{I}$ pairs have much lower

A

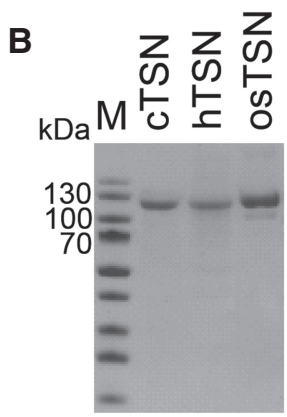

C

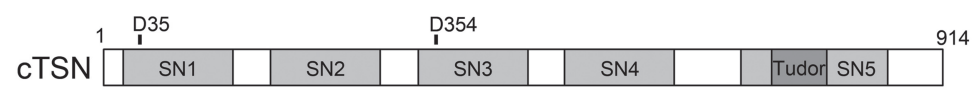

hTSN
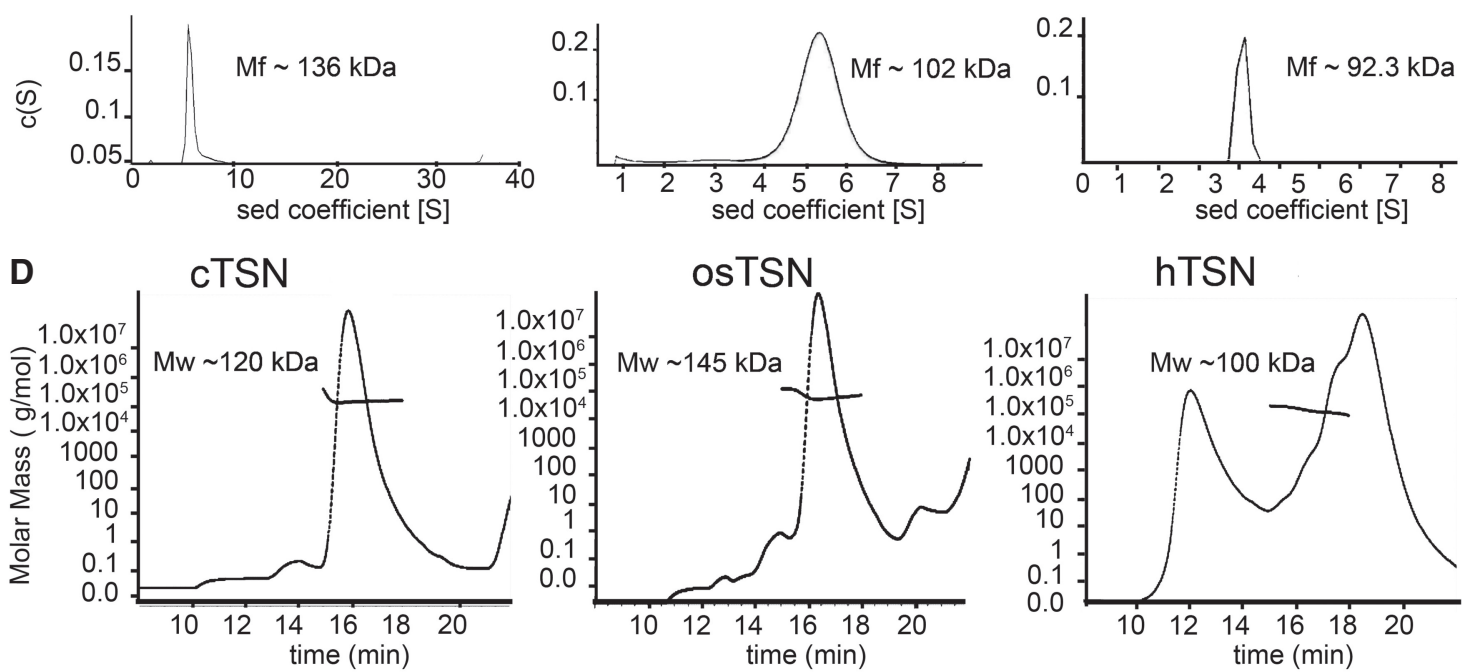

FIGURE 1. Full-length TSN is a monomeric protein. (A) The domain structure of Caenorhabditis elegans cTSN. The two putative Ca ${ }^{2+}$-binding residues in the SN1 and SN3 domains are shown at the top. (B) The SDS-PAGE of the purified recombinant cTSN from Caenorhabditis elegans, hTSN from Homo sapiens, and osTSN from Oryza sativa. $(C)$ The molecular weights of cTSN, osTSN, and hTSN were estimated by ultracentrifugation. (D) TSN proteins were subjected to SEC-MALS (size-exclusion chromatography coupled with multiangle light scattering) and are represented as elution profiles. 
thermal stability compared to those with Watson-Crick base pairs (Serra et al. 2004). This finding prompted us to examine if TSN cleaves dsRNA with mismatched base pairs. Here, we show that Caenorhabditis elegans TSN (cTSN) degrades both ssRNA and mismatched dsRNA, but not perfectly matched dsRNA. Therefore, TSN targets the unstructured regions of RNA, but not necessarily those with inosines or $\mathrm{I} \cdot \mathrm{U} / \mathrm{U} \cdot \mathrm{I}$ pairs. We also identified multiple ribonuclease active sites in the SN1 and SN3 domains of cTSN and show by small-angle X-ray scattering (SAXS) that rice TSN (osTSN) has a flexible two-lobed conformation. Together, our results reveal how TSN selects its targets for degradation and explain how TSN degrades inosine-edited miRNA precursors and mature miRNA during miRNA decay.

\section{RESULTS}

\section{TSN is a monomeric protein}

We first inserted the cDNA encoding TSN from worms (Caenorhabditis elegans), plants (Oryza sativa), and mammals (Homo sapiens) into the expression vectors to express TSN proteins with an N-terminal His-tag in E. coli. Human, rice, and C. elegans TSN, named hTSN, osTSN, and cTSN, respectively, were purified by chromatography with a high homogeneity (Fig. 1B). The molecular weights of TSN were estimated by analytical ultracentrifugation (AUC): $136 \mathrm{kDa}$ for cTSN (calculated MW: $101 \mathrm{kDa}$ ), $102 \mathrm{kDa}$ for osTSN (calculated MW: $108 \mathrm{kDa}$ ), and $92 \mathrm{kDa}$ for hTSN (calculated MW: 102 $\mathrm{kDa}$ ) (Fig. 1C). Similar molecular weights- $120 \mathrm{kDa}$ for cTSN, $145 \mathrm{kDa}$ for osTSN, and $100 \mathrm{kDa}$ for hTSN-were estimated for TSN proteins using SEC-MALS (size-exclusion chromatography coupled with multiangle light scattering) (Fig. 1D). These results suggest that these three recombinant TSN proteins are monomeric in solution. However, hTSN and cTSN were expressed at low levels in E. coli, and hTSN was highly unstable, tending to decay and aggregate (see Fig. 1D). In contrast, osTSN was highly expressed and could be purified in large quantities. As a result, we continued our RNA degradation assays using cTSN since it shares a higher sequence identity of $47 \%$ with hTSN, but performed SAXS (small-angle X-ray diffraction) using osTSN, which has a lower sequence identity of $33 \%$ with hTSN.

\section{cTSN is a $\mathrm{Ca}^{2+}$-dependent RNase cleaving at the $5^{\prime}$-side of a phosphodiester bond}

As the endonuclease activity of hTSN is promoted by calcium ions (Elbarbary et al. 2017b), we first examined the metal ion-dependent activity of cTSN. We prepared a stem-loop RNA, named pre-miR142, which shares a similar structure and sequence to the pre-miR142 precursor that was used in a previous report (Yang et al. 2006). cTSN was incubated with the $5^{\prime}$-end fluorescein-labeled pre-miR142 in buffers containing different metal ions, and the digested RNA fragments were analyzed by gel electrophoresis (Fig. 2A). The purified cTSN could degrade RNA before metal ions were added and this activity was inhibited by $100 \mu \mathrm{M}$ EGTA, suggesting that the recombinant cTSN was bound with endogenous metal ions (see the first three lanes in Fig. 2A). To remove the endogenous metal ions, cTSN was pre-treated with $20 \mu \mathrm{M}$ EGTA prior to the RNA degradation assays. The EGTA-treated cTSN had almost no RNase activity, suggesting that the endogenous metal ions had been mostly removed. Addition of calcium ions promoted cTSN endonuclease activity at optimal concentrations of 0.1 to $1 \mathrm{mM}$ (Fig. 2A). However, cTSN had only residual endonuclease activity with $1 \mathrm{mM}$ magnesium ions and no activity with $1 \mathrm{mM}$ zinc ions. Together, these results suggest that cTSN is a $\mathrm{Ca}^{2+}$-dependent endonuclease in RNA degradation, similar to the bacterial homolog staphylococcal nuclease (Cotton et al. 1979).
A

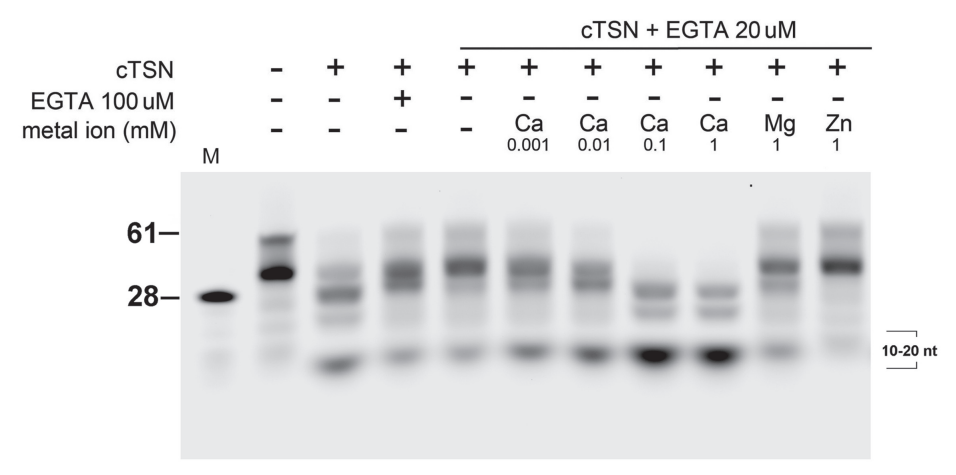

B

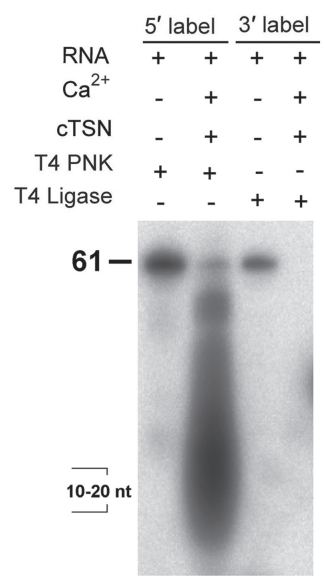

FIGURE 2. cTSN is a $\mathrm{Ca}^{2+}$-dependent endonuclease cleaving at the $5^{\prime}$-side of phosphodiester bonds. (A) cTSN ( $\left.100 \mathrm{nM}\right)$ degraded the $5^{\prime}$-fluoresceinlabeled pre-miR142 RNA $(500 \mathrm{nM})$ in the presence of $\mathrm{Ca}^{2+}$ at concentrations of $0.1-1 \mathrm{mM}$. The sizes of pre-miR142 (68 nt) and an RNA marker (28 $\mathrm{nt}$ ) were labeled in the left of the gel. (B) cTSN cleaved at the $5^{\prime}$-side of phosphodiester bonds to produce degraded fragments with $3^{\prime}$-phosphate and $5^{\prime}$ $\mathrm{OH}$ ends that could be labeled by T4 polynucleotide kinase (T4 PNK) but not by T4 RNA ligase (T4 ligase). 
To examine the products cleaved by cTSN, the digested RNA was further incubated with two different end-labeling enzymes: T4 polynucleotide kinase (T4 PNK) that only adds $\mathrm{P}^{32}$-labeled-phosphates to $5^{\prime}$ hydroxyl groups of RNA chains, and T4 RNA ligase that only adds $\mathrm{P}^{32}$-labeled-phosphates to the $3^{\prime}$ hydroxyl groups. The labeling results clearly show that the cleaved RNA fragments could only be labeled by T4 PNK and not by T4 ligase, suggesting that the digested products had 5'-hydroxyl and 3'-phosphate ends (Fig. 2B). In summary, cTSN is a $\mathrm{Ca}^{2+}$-dependent ribonuclease that conducts endonucleolytic cleavage at the $5^{\prime}$ site of the phosphodiester bonds of RNA chains.

\section{cTSN preferentially binds double-stranded RNA harboring inosines}

Human TSN degrades A-to-I-edited primary miRNA precursors, including pre-miR142 (Yang et al. 2006). Next, we tested if cTSN preferentially binds to the A-to-I-edited pre-miR142 by filter-binding assays. We prepared a $5^{\prime}$ end $\mathrm{P}^{32}$-labeled stem-loop RNA mimicking the primary
miR142 precursor but in which the Dicer-cleavable loop region was replaced with a short loop (referred to as premiR142), as well as its inosine-edited counterpart (termed pre-miR142-AtoI) (see the RNA sequences in Fig. 3). We found that A-to-I editing indeed improved the binding affinity between cTSN and RNA, with a twofold greater $K_{\mathrm{d}}$ of $74.6 \pm 3.3 \mathrm{nM}$ for the edited pre-miR142-AtoI compared to a $K_{\mathrm{d}}$ of $135.9 \pm 16.1 \mathrm{nM}$ for the unedited premiR142. We also generated unedited and edited RNA lacking the short loop region, named pre-miR142 $\Delta$ loop and premiR142 $\Delta$ loop-AtoI, respectively. A-to-I editing again increased the $K_{\mathrm{d}}$ value (about fourfold) of cTSN and RNA-binding affinity; a $K_{\mathrm{d}}$ of $235.8 \pm 38.2 \mathrm{nM}$ for unedited pre-miR142 $\Delta$ loop and $68.3 \pm 7.1 \mathrm{nM}$ for edited premiR142 $\Delta$ loop-AtoI, respectively. cTSN bound the RNA with the loop (pre-miR142) more strongly (twofold) than the RNA lacking the loop (pre-miR142 $\Delta$ loop) (Fig. 2). Taken together, these results show that A-to-I editing increased binding affinity two- to fourfold, whereas the 5-nucleotide (nt) loop region increased the binding affinity of RNA to cTSN twofold.

\begin{tabular}{|c|c|c|c|}
\hline & RNA & Sequence & $\mathrm{Kd} \mathrm{nM}\left(\mathrm{R}^{2}\right)$ \\
\hline & pre-miR142 & $\begin{array}{l}\text { 5'. CAGUCACCCAUAAAGUAGAAAGCACUACU } U^{G} \\
\text { GUGAGUAGGUAUUUCAUCCUUUGUGAUG } \\
\text { GG }\end{array}$ & $135.9 \pm 16.1(0.97)$ \\
\hline & pre-miR142-Atol & $\begin{array}{l}\text { 5'-CAGUCACCCAUU\|AGU|GAAAGCACUAC U } \\
\text { GUGAGUAGGGUIUUUCIUCCUUUGUGIUG } \\
\text { GUG }\end{array}$ & $74.6 \pm 3.3(0.99)$ \\
\hline & pre-miR142 $\Delta$ loop & $\begin{array}{l}\text { 5'. CAGUCACCCAUAAAGUAGAAAGCACUAC } \\
\text { GUGAGUÜGGUAUUUCAUCCOUUUGGUGAUG }\end{array}$ & $235.8 \pm 38.2(0.96)$ \\
\hline & pre-miR142 $\Delta$ loop-Atol & $\begin{array}{l}\text { 5'- CAGUCACCCAU||AGU|GAAAGCACUAC } \\
\text { GUGAGUAGGGU|UUUCIUCGUUUQGGGIUG }\end{array}$ & $68.3 \pm 7.1(0.97)$ \\
\hline & dsRNA & $\begin{array}{l}\text { 5'-ACUGGACAAAUACUCCGAGG } \\
\text { UGACCUGUUUAUGAGGCUCC }\end{array}$ & $392.9 \pm 51.5(0.97)$ \\
\hline ताTाTाT & ssRNA & 5'-ACUGGACAAAUACUCCGAGG & $767.3 \pm 131.2(0.96)$ \\
\hline
\end{tabular}

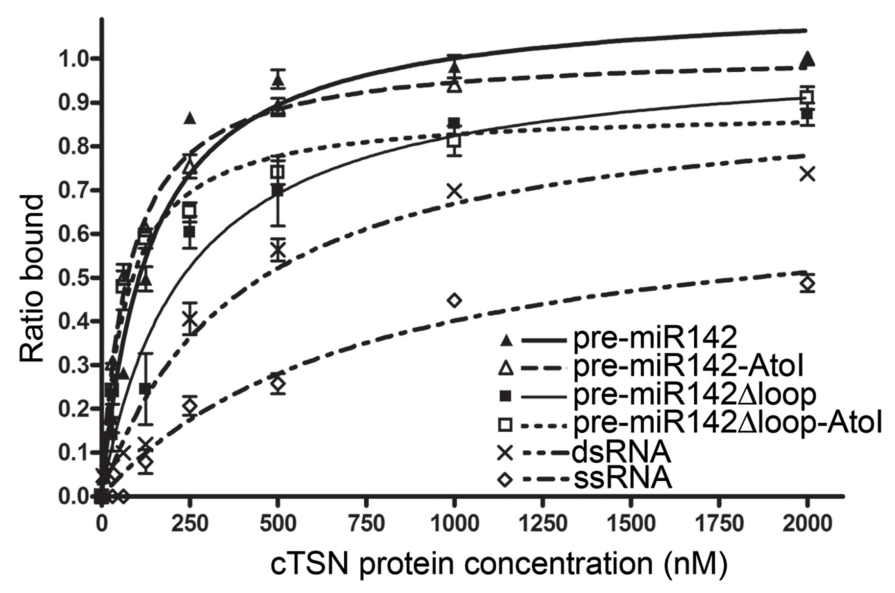

FIGURE 3. cTSN preferentially binds double-stranded RNA containing $\mathrm{I} \cdot \mathrm{U}$ and $\mathrm{U} \cdot \mathrm{I}$ pairs. cTSN $(0-2.0 \mu \mathrm{M})$ was incubated with $5^{\prime}-\mathrm{P}^{32}$-labeled RNA for filter-binding assays. Binding percentages from three measurements were calculated to derive the apparent $K_{\mathrm{d}}$ values between cTSN and RNA by a one-site binding curve, with $R^{2}$ (all greater than 0.95) shown in parentheses. 
To further clarify if cTSN prefers to target the ssRNA or ssRNA region imbedded in dsRNA, we also measured the binding affinity between cTSN and a 20-bp perfectly matched dsRNA (dsRNA) versus a 20-nt ssRNA (ssRNA). cTSN bound the dsRNA with an approximately twofold higher affinity $(392.9 \pm 51.5 \mathrm{nM})$ than the ssRNA $(767.3 \pm 132 \mathrm{nM})$, suggesting that cTSN prefers to bind dsRNA than ssRNA (see Fig. 3 ). Taken together, these results suggest that cTSN preferentially binds double-stranded RNA possessing inosines or loops.

\section{cTSN preferentially cleaves ssRNA and the unstructured regions of dsRNA}

Next, we wondered if cTSN preferentially degrades A-to-I-edited RNA. We incubated the $5^{\prime}$-end fluorescein-labeled premiR142 with cTSN and the RNA cleavage patterns were re- vealed by gel electrophoresis (see Fig. 4A). cTSN degraded the edited pre-miR142AtoI (7\% substrate remained) better than its unedited counterpart (pre-miR142, 25\% substrate remained). Similarly, cTSN degraded the edited premiR142 $\Delta$ loop-AtoI ( $6 \%$ substrate remained) better than the unedited pre-miRNA142 $\Delta$ loop (58\% substrate remained). These results are consistent with those of our binding assays and show that inosine editing of miRNA precursors indeed enhances degradation by cTSN.

A-to-I editing introduces unstable $\mathrm{I} \cdot \mathrm{U}$ or $\mathrm{U} \cdot \mathrm{I}$ base pairs, suggesting that this type of base-pairing induces structural changes (Serra et al. 2004). Therefore, we set out to examine if cTSN recognizes inosines or mismatched regions. We prepared 20-nt unedited and edited RNAs, referred to, respectively, as ssRNA and ssRNA-AtoI, containing either AAUA or IIUI in the middle of the chain, i.e., similar to those used

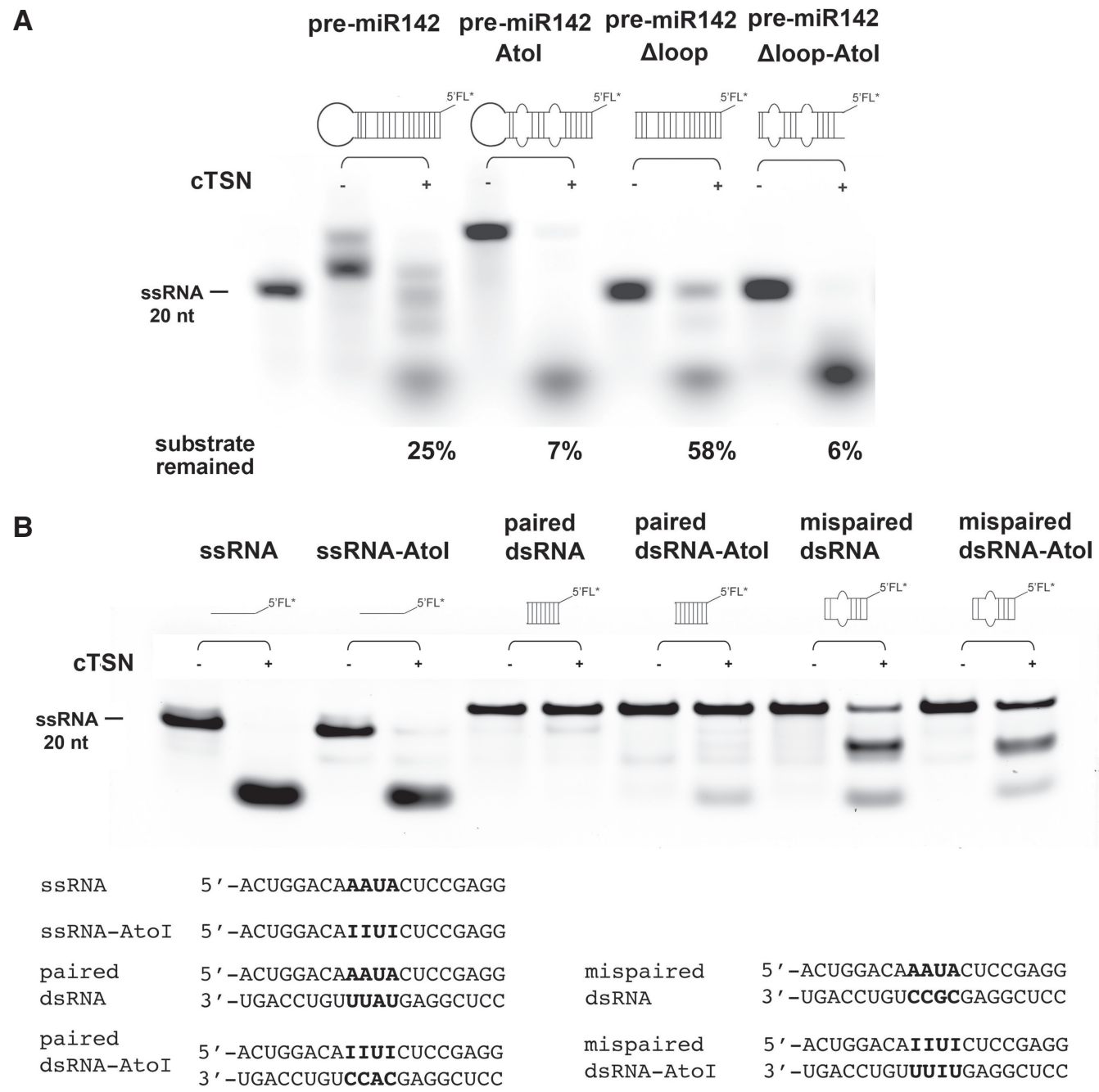

FIGURE 4. cTSN degrades single-stranded RNA and mismatched double-stranded RNA with or without inosines. $(A)$ cTSN (100 nM) degraded stem-loop 5'-fluorescein-labeled RNAs $(500 \mathrm{nM})$ containing mispaired or loop regions (the percentages of the remained substrates are listed at the bottom of the gel). (B) cTSN (100 nM) degraded single-stranded RNA (500 nM) with or without inosines, as well as double-stranded RNA containing mispaired base pairs, including AAUC/CGCC and IIUI/UIUU. In contrast, cTSN could not degrade paired double-stranded RNA with or without inosines. The RNA sequences are listed at the bottom of the gel. 
in a previous analysis (Scadden 2005). We also prepared perfectly paired 20-bp dsRNAs, referred to as "paired dsRNA" and "paired dsRNA-AtoI," containing either $A \cdot \mathrm{U}$ base pairs (AAUA/UAUU) or $\mathrm{I} \cdot \mathrm{C}$ base pairs (IIUI/CACC) in the middle of the duplex respectively, since inosine (I) and cytosine (C) can form a stable $\mathrm{I} \cdot \mathrm{C}$ base pair (Watkins and SantaLucia 2005). Our degradation assays show that cTSN degraded ssRNA with or without inosines (i.e., ssRNA and ssRNA-AtoI), but could not degrade the perfectly paired dsRNAs with or without inosines (i.e., paired RNA or paired dsRNA-AtoI) (see Fig. 4B). These results demonstrate that the presence of inosines in RNA is not the determining factor for RNA degradation by cTSN.

We next prepared two mispaired dsRNAs with or without inosines in the middle of the chains: "mispaired dsRNA" containing AAUA/CGCC, and "mispaired dsRNA-AtoI" containing IIUI/UIUU. Notably, cTSN degraded both mispaired dsRNAs, i.e., with or without inosines (Fig. 4B). These findings suggest that, besides ssRNA, cTSN preferentially cleaves dsRNA containing mismatched regions, whether or not they contain inosines. Since A-to-I editing alters the stability of base-pairing in dsRNA, we conclude that the enhanced degradation of inosine-edited RNA by TSN is due to structural changes in the RNA. In summary, our results suggest that cTSN is not an inosine-specific ribonuclease, but instead it preferentially degrades ssRNA and dsRNA containing unstructured mismatched regions.

\section{Critical catalytic residues in cTSN are located in the $\mathrm{SN} 1$ and SN3 domains}

Structure-based sequence alignment shows that most putative catalytic residues are mutated in the $\mathrm{SN}$ domains of TSN when compared with those of staphylococcal nuclease (Li et al. 2008). The metal ion-binding residues in domains SN2, SN4, and SN5 of TSN proteins are degenerated, whereas one of the calcium-binding residues is conserved in $\mathrm{SN} 1$ (Asp35) and SN3 (Asp354) in cTSN (Fig. 5A). To clarify which SN domain is responsible for cTSN's endonuclease activity, we constructed single-point mutants of cTSN, D35A, and D354A, and the double-point mutant D35A/D354A for endonuclease activity assays. With long degradation time $(3 \mathrm{~h})$, the wild-type cTSN cleaved pre-miR142 and premiR142AtoI into small RNA fragments of about 10-20 nt (Fig. 5B). The single-point mutants D35A and D354A de- graded the $5^{\prime}$-end fluorescein-labeled inosine-containing pre-miR142-AtoI comparably to wild-type cTSN (Fig. 5C). In contrast, the double-point mutant D35A/D354A exhibited dramatically reduced endonuclease activity in our timecourse assays (Fig. 5C). These results suggest that inactivation of one of the SN domains cannot impair cTSN activity, with the protein likely having at least two active sites in the SN1 and SN3 domains. Our findings agree with the sequence alignment results, which suggest that $\mathrm{SN} 1$ and $\mathrm{SN} 3$ likely bear endonuclease activities ( $\mathrm{Li}$ et al. 2008). However, they are not consistent with a truncation study showing that SN1 and SN4 in hTSN are crucial for endonuclease activity (Elbarbary et al. 2017b) (see Discussion).

\section{Overall structure of TSN}

Although the crystal structures of SN1-SN2 and SN3-SN4Tudor-SN5 of hTSN were previously reported (Li et al. 2008; Guo et al. 2014), it remains unclear how full-length TSN folds and binds to RNA. Therefore, we performed SAXS (small-angle light scattering) to determine the overall folding of TSN in solution. Only osTSN produced SAXS data of a reasonable quality, but the distance distribution functions show characteristic smoothing toward 0 as $r$ approaches $D_{\max }$ around $191 \AA$ (Fig. 6A), suggesting a flexible elongated conformation as reported previously from FRET 

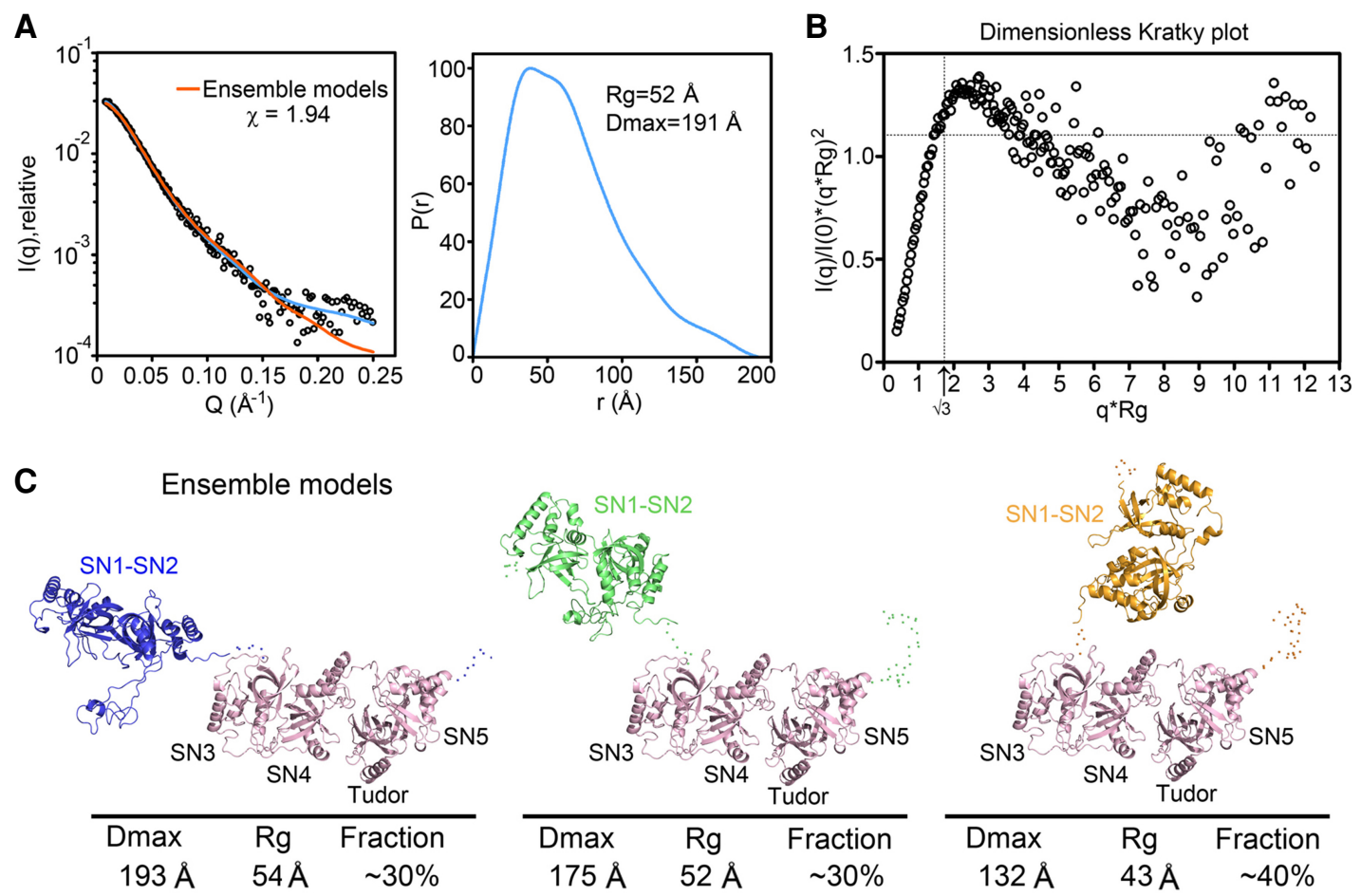

FIGURE 6. Solution SAXS analysis of rice osTSN reveals a flexible two-lobed structure. (A) Small-angle X-ray scattering (SAXS) curves are represented as logarithmic scattering intensities. Theoretical scattering intensities generated from ensemble models are fitted to experimental data with a $\chi$ value of 1.94. Right panel is the distance distribution function with a maximum diameter $\left(D_{\max }\right)$ of $191 \AA$ and a radius of gyration $\left(R_{\mathrm{g}}\right)$ of $52 \AA$. $(B)$ The $R_{\mathrm{g}}$-based dimensionless Kratky plot with curve peak at a $q \times R_{\mathrm{g}}$ of greater than $\sqrt{3}$, indicating flexible conformations. $(C)$ Three major ensemble models, with the number of times each conformation existed within the ensemble represented as fractions (\%) below each conformation.

analysis (Guo et al. 2014). We calculated the $R_{\mathrm{g}}$-based dimensionless Kratky plot to reveal that osTSN exhibits some degree of flexibility (Fig. 6B; Receveur-Brechot and Durand 2012). We next modeled the structure of osTSN by the ensemble optimization method (Petoukhov et al. 2012) using the two crystal structures of SN1-SN2 (Guo et al. 2014) and SN3-SN4-Tudor-SN5 (Li et al. 2008) as two rigid bodies in the ensemble search. Three major osTSN conformations were generated and the ensemble gave a fitting curve to the experimental SAXS data with an $\chi$ value of 1.94 (Fig. 6A). Two conformations, each representing $\sim 30 \%$ of the ensemble structures, exhibited a "wide open" format with a $D_{\max }$ of $193 \AA$ and a "half open" conformation with a $D_{\max }$ of $175 \AA$, respectively. One conformation, representing $\sim 40 \%$ of the structures, exhibited a "closed" conformation with a $D_{\max }$ of $132 \AA$ (Fig. 6C). Thus, our SAXS analyses demonstrate that osTSN exhibits a flexible two-lobed conformation in which SN1-SN2 can switch from an open to a closed conformation relative to SN3-SN4-Tudor-SN5. In summary, our SAXS results suggest that TSN has a flexible elongated structure that may change conformation upon RNA binding.

\section{DISCUSSION}

In this study, we show that cTSN is a $\mathrm{Ca}^{2+}$-dependent ribonuclease, degrading not only single-stranded RNA but also
dsRNA containing mismatched regions arising from A-to-I editing that produces multiple $\mathrm{I} \cdot \mathrm{U}$ and $\mathrm{U} \cdot \mathrm{I}$ pairs or from mismatched base-pairing, such as the $A \cdot C$ and $U \cdot G$ pairs used here. Perfectly paired dsRNA substrates with or without inosines are resistant to cTSN cleavage. An inosine-specific ribonuclease (Endo V) has previously been characterized (Morita et al. 2013), and we conclude that TSN is not inosine-specific but is a structure-specific ribonuclease that targets singlestranded RNA and dsRNA with loose regions. Presence of long perfect dsRNA molecules in the cell usually indicates viral infection and invading nucleic acids. TSN may work with ADAR that hyper-edits long dsRNA so that these molecules can be further degraded by TSN in cellular defense (Samuel 2011).

Another important function of TSN is miRNA decay. It has been shown that TSN degrades certain hyper-edited primary miRNA precursors (Yang et al. 2006), as well as a group of mature miRNAs with a preference to cleave at UA and CA dinucleotides (Elbarbary et al. 2017b). Inosine editing may loosen the base-pairing of miRNA precursors and increase their exposure to degradation by TSN. A recent study further shows that TSN interacts with UPF1 helicase which can dissociate miRNA from their mRNA targets, making the mature single-stranded miRNAs susceptible to the TSN-mediated miRNA decay (TumiD) (Elbarbary et al. 2017a). Our results thus reveal how TSN degrades inosine-edited primary 
miRNA precursors, as well as mature miRNAs, playing an important role in miRNA decay.

TSN is a highly conserved protein and contains five staphylococcal nuclease-like (SN) domains. However, compared with its bacterial homolog, most catalytic residues in the $\mathrm{SN}$ domains are degenerated. In this study, we show that $C$. elegans cTSN contains at least two active sites located in domains SN1 and SN3. Interestingly, the putative $\mathrm{Ca}^{2+}$-binding residue Asp354 in the $\mathrm{SN} 3$ domain is strictly conserved among TSN proteins, but Asp35 in the SN1 domain is only conserved in protozoa, plants, and invertebrates and not in vertebrates. A recent study has shown that the SN1 and SN4 domains in hTSN are important for TSN's catalytic activity (Elbarbary et al. 2017b). As SN1-SN2 and SN3-SN4 are assembled, respectively, into a structural lobe (see Fig. 6), deletion of SN1 or SN4 might disrupt the folding of each lobe and impair TSN's ribonuclease activity, similar to our results of mutations in SN1 and SN3 domains. Further investigations are required to identify the active $\mathrm{SN}$ domains in human TSN, which will be important for designing TSN inhibitors in anti-cancer therapy.

\section{MATERIALS AND METHODS}

\section{Protein expression and purification}

The full-length cDNA of Caenorhabditis elegans was cloned by RTPCR. The cDNA of Homo sapiens TSN was purchased from OpenBiosystems, whereas the cDNA of Oryza sativa TSN was purchased from the KOME database. The cDNA of each TSN was inserted into pET28a expression vector using XhoI and NdeI restriction cutting sites to express proteins with C-terminal 6xHistidine tags in E. coli. Site-directed mutagenesis constructs of cTSN were prepared by Pfu-Ultra II Fusion HS DNA Polymerase kit (Agilent Technologies). All constructs were expressed in E. coli BL21 (DE3) RIPL cells (Agilent Technologies) at an $\mathrm{OD}_{600}$ of 0.6 with $0.5 \mathrm{mM}$ IPTG for $16 \mathrm{~h}$ at $18^{\circ} \mathrm{C}$. Cells were harvested and lysed at $4^{\circ} \mathrm{C}$ in $50 \mathrm{mM}$ HEPES ( $\mathrm{pH} 7.0$ ), $150 \mathrm{mM} \mathrm{NaCl}, 10 \mathrm{mM}$ imidazole, $10 \%$ glycerol, $0.1 \mathrm{mM}$ PMSF, $5 \mathrm{mM} \beta$-Mercaptoethanol, and $0.1 \%$ CHAPS by microfluidizer (Microfluidics M-110P). Cell lysate was passed through a His-Trap HP column (GE HealthCare) and TSN proteins were eluted with $50 \mathrm{mM}$ HEPES ( $\mathrm{pH} 7.0$ ), $150 \mathrm{mM}$ $\mathrm{NaCl}, 250 \mathrm{mM}$ imidazole, and $5 \%$ glycerol. TSN protein samples were then passed through a HiTrap Heparin HP column (GE HealthCare) and eluted with a gradient of 0 to $1 \mathrm{M} \mathrm{NaCl}$ in PBS buffer ( $\mathrm{pH}$ 7.4). Eluted protein samples were adjusted to a concentration of $2 \mathrm{mg} / \mathrm{mL}$ in PBS buffer ( $\mathrm{pH}$ 7.4) with addition of $150 \mathrm{mM}$ $\mathrm{NaCl}$ as stock for further experiments.

\section{Ultracentrifugation}

For AUC assays, the recombinant TSN proteins were concentrated to an $\mathrm{OD}_{280}$ of 1.0 in $50 \mathrm{mM}$ HEPES (pH 7.0), $150 \mathrm{mM} \mathrm{NaCl}, 10 \mathrm{mM}$ imidazole, and $1 \mathrm{mM} \beta$-mercaptoethanol. Sedimentation velocities of TSN were measured at 40,000 rpm (Beckman XL-A) at $4^{\circ} \mathrm{C}$. Multiple scans $\left(\mathrm{OD}_{280}\right)$ at different time intervals were then fitted to a continuous $\mathrm{c}(\mathrm{s})$ distribution model using the SEDFIT program.

\section{SEC-MALS}

TSN ( $1 \mathrm{mg} / \mathrm{mL}, 100 \mu \mathrm{L}$ injection volume) was applied to an AgilentBio SEC-3 column (Agilent) connected to a DAWN HELIOS II-18 angle MALS (Wyatt Technology) detector with a wavelength setting at $658 \mathrm{~nm}$. Samples were run in a PBS ( $\mathrm{pH} 7.4$ ) buffer containing $150 \mathrm{mM} \mathrm{NaCl}$ and $5 \mathrm{mM} \beta$-mercaptoethanol, with a flow rate of $0.2 \mathrm{~mL} / \mathrm{min}$ using the ÄKTA-UPC 900 FPLC system (GE Healthcare). A $2 \mathrm{mg} / \mathrm{mL}$ sample of BSA (Sigma Lot\#SLBH1159V) was used for calibration. Scattering data were analyzed using the ASTRA 6 software (Wyatt Technology).

\section{RNA terminal labeling experiments}

The pre-miR142 degraded by cTSN was used for terminal labeling assays. For $5^{\prime}$-end labeling, degraded RNA fragments, $\gamma$ - $\mathrm{P}^{32}$-ATP and $\mathrm{T} 4$ polynucleotide kinase (Roche) were mixed and incubated at $37^{\circ} \mathrm{C}$ for $30 \mathrm{~min}$. For $3^{\prime}$-end labeling, degraded RNA fragments, a- $\mathrm{P}^{32} \mathrm{ATP}$ and T4 RNA ligase (NEB) were mixed and incubated at $37^{\circ} \mathrm{C}$ for $30 \mathrm{~min}$. Reactions were stopped by sample loading buffers and the labeling results were analyzed on 15\% TBE-PAGE.

\section{Filter-binding assays}

The $5^{\prime}$-end ${ }^{32} \mathrm{P}$-labeled RNA substrates shown in Figure 3 (purchased from Dharmacon) were incubated with a serial dilution of CTSN ( 0 to $2 \mu \mathrm{M}$ ) in binding buffer containing $50 \mathrm{mM}$ HEPES ( $\mathrm{pH} 7.0$ ), 250 $\mathrm{mM} \mathrm{NaCl}, 5 \mathrm{mM} \beta$-Mercaptoethanol and $2 \mathrm{mM}$ EDTA for $30 \mathrm{~min}$ at room temperature. The cTSN-RNA complex solutions were passed through a filter-binding assay device (Bio-Dot SF microfiltration apparatus, Bio-Rad). The isotope signals from cTSN-RNA complex bound on the nitrocellulose membrane and the free RNA passed through the filter-binding assay apparatus were exposed to a phosphorimaging plate and visualized by autoradiography (Fujifilm, FLA-5000). The intensities of cTSN-RNA complex and free RNA were quantified and analyzed by GraphPad Prism 4. Binding percentages from three measurements were calculated to derive the apparent $K_{\mathrm{d}}$ between cTSN and RNA by a one-site binding curve fitting using the equation $Y=X /\left(K_{\mathrm{d}}+X\right)$, where $X$ is the cTSN concentration and $Y$ is the percentage of bound RNA/total RNA.

\section{RNA degradation assays}

For the RNA degradation experiments shown in Figure 2A, premiR142 (sequences listed in Fig. 3) was labeled at the $5^{\prime}$ end with fluorescein (Dharmacon). The $5^{\prime}$-end fluorescein-labeled premiR142 (500 nM) was incubated with cTSN $(100 \mathrm{nM})$ in reaction buffer containing $50 \mathrm{mM}$ Tris- $\mathrm{HCl}$ ( $\mathrm{pH} 7.6$ ), $100 \mathrm{mM} \mathrm{NaCl}, 5 \%$ glycerol, $0.01 \%$ CHAPS, and $5 \mathrm{mM} \beta$-mercaptoethanol at $25^{\circ} \mathrm{C}$ for $1 \mathrm{~h}$ with or without the presence of different metal ions. The RNA degradation results were analyzed by $10 \%$ TBE-PAGE.

For the RNA degradation experiments shown in Figures 4 and 5, the $5^{\prime}$-fluorescein-labeled RNA $(500 \mathrm{nM})$ was incubated with cTSN $(100 \mathrm{nM})$ or the cTSN mutants $(100 \mathrm{nM})$ in reaction buffer containing $50 \mathrm{mM}$ Tris- $\mathrm{HCl}$ (pH 7.6), $100 \mathrm{mM} \mathrm{NaCl}, 5 \%$ glycerol, $0.01 \%$ CHAPS, $5 \mathrm{mM} \beta$-mercaptoethanol, and $1 \mathrm{mM} \mathrm{CaCl}_{2}$ at $25^{\circ} \mathrm{C}$ for $1 \mathrm{~h}$ (longer incubation time of $3 \mathrm{~h}$ was used for Fig. 5B). The cleavage results were analyzed by $10 \%$ or $15 \%$ TBE-PAGE. 


\section{SAXS}

Small-angle X-ray scattering data were recorded at $15^{\circ} \mathrm{C}$ at the SAXS beamline 23A NSRRC in Hsinchu, Taiwan. The osTSN protein sample $(1 \mathrm{mg} / \mathrm{mL}$ in PBS buffer containing an additional $150 \mathrm{mM} \mathrm{NaCl}$ and $5 \mathrm{mM} \beta$-mercaptoethanol at $\mathrm{pH}$ 7.4) was injected into an Agilent-Bio SEC-3 column at a flow rate of $0.02 \mathrm{~mL} / \mathrm{min}$. An X-ray wavelength of $0.8266 \AA$ was used for data collection. Selected frames were merged and analyzed for initial $R_{\mathrm{g}}$ estimation by the PRIMUS program (Konarev et al. 2003), and then by the GNOM program (Svergun 1992) for $D_{\max }$ and $P(r)$ distance distribution (ATSAS program suite, version 2.7) (Petoukhov et al. 2012). Computation of the ensemble of osTSN was conducted using the Ensemble Optimization Method using ATSAS online (Tria et al. 2015).

\section{ACKNOWLEDGMENTS}

This work was supported by Academia Sinica and Ministry of Science and Technology, Taiwan, R.O.C. Portions of this research were carried out at the National Synchrotron Radiation Research Center, a national user facility supported by the Ministry of Science and Technology of Taiwan.

Received October 16, 2017; accepted February 12, 2018.

\section{REFERENCES}

Abe S, Wang PL, Takahashi F, Sasaki E. 2005. Structural analysis of cDNAs coding for $4 \mathrm{SNc}$-Tudor domain protein from fish and their expression in yellowtail organs. Marine Biotechnol (NY) 7: 677-686.

Blanco MA, Alečković M, Hua Y, Li T, Wei Y, Xu Z, Cristea IM, Kang Y. 2011. Identification of staphylococcal nuclease domain-containing 1 (SND1) as a Metadherin-interacting protein with metastasis-promoting functions. J Biol Chem 286: 19982-19992.

Callebaut I, Mornon JP. 1997. The human EBNA-2 coactivator p100: multidomain organization and relationship to the staphylococcal nuclease fold and to the tudor protein involved in Drosophila melanogaster development. Biochem J 321: 125-132.

Cappellari M, Bielli P, Paronetto MP, Ciccosanti F, Fimia GM, Saarikettu J, Silvennoinen O, Sette C. 2014. The transcriptional co-activator SND1 is a novel regulator of alternative splicing in prostate cancer cells. Oncogene 33: 3794-3802.

Caudy AA, Ketting RF, Hammond SM, Denli AM, Bathoorn AM, Tops BB, Silva JM, Myers MM, Hannon GJ, Plasterk RH. 2003. A micrococcal nuclease homologue in RNAi effector complexes. Nature 425: 411-414.

Cotton FA, Hazen EEJ, Legg MJ. 1979. Staphylococcal nuclease: Proposed mechanism of action based on structure of enzyme-thymidine $3^{\prime}, 5^{\prime}$-bisphosphate-calcium ion complex at $1.5-\AA$ resolution. Proc Natl Acad Sci 76: 2551-2555.

Elbarbary RA, Miyoshi K, Hedaya O, Myers JR, Maquat LE. 2017a. UPF1 helicase promotes TSN-mediated miRNA decay. Genes Dev 31: 1483-1493.

Elbarbary RA, Miyoshi K, Myers JR, Du P, Ashton JM, Tian B, Maquat LE. 2017b. Tudor-SN-mediated endonucleolytic decay of human-cell microRNAs promotes G1/S phase transition. Science 356: 859-862.

Emdad L, Janjic A, Alzubi MA, Hu B, Santhekadur PK, Menezes ME, Shen XN, Das SK, Sarkar D, Fisher PB. 2015. Suppression of miR184 in malignant gliomas upregulates SND1 and promotes tumor aggressiveness. Neuro Oncol 17: 419-429.

Frei dit Frey N, Muller P, Jammes F, Kizis D, Leung J, PerrotRechenmann C, Bianchi MW. 2010. The RNA binding protein Tudor-SN is essential for stress tolerance and stabilizes levels of stress-responsive mRNAs encoding secreted proteins in Arabidopsis. Plant Cell 22: 1575-1591.

Friberg A, Corsini L, Mourão A, Sattler M. 2009. Structure and ligand binding of the extended Tudor domain of D. melanogaster TudorSN. J Mol Biol 387: 921-934.

Gao X, Zhao X, Zhu Y, He J, Shao J, Su C, Zhang Y, Zhang W, Saarikettu J, Silvennoinen O, et al. 2012. Tudor staphylococcal nuclease (Tudor-SN) participates in small ribonucleoprotein (snRNP) assembly via interacting with symmetrically dimethylated Sm proteins. J Biol Chem 287: 18130-18141.

Guo F, Wan L, Zheng A, Stanevich V, Wei Y, Satyshur KA, Shen M, Lee W, Kang Y, Xing Y. 2014. Structural insights into the tumor-promoting function of the MTDH-SND1 complex. Cell Rep 8: 1704-1713.

Gutierrez-Beltran E, Moschou PN, Smertenko AP, Bozhkov PV. 2015. Tudor staphylococcal nuclease links formation of stress granules and processing bodies with mRNA catabolism in Arabidopsis. Plant Cell 27: 926-943.

Gutierrez-Beltran E, Denisenko TV, Zhivotovsky B, Bozhkov PV. 2016. Tudor staphylococcal nuclease: biochemistry and functions. Cell Death Differ 23: 1739-1748.

Konarev PV, Volkov VV, Sokolova AV, Koch MHJ, Svergun DI. 2003. PRIMUS: a Windows PC-based system for small-angle scattering data analysis. J Appl Cryst 36: 1277-1282.

Kuruma H, Kamata Y, Takahashi H, Igarashi K, Kimura T, Miki K, Miki J, Sasaki H, Hayashi N, Egawa S. 2009. Staphylococcal nuclease domain-containing protein 1 as a potential tissue marker for prostate cancer. Am J Pathol 174: 2044-2050.

Leverson J, Koskinen PJ, Orrico F, Rainio EM, Jalkanen KJ, Dash AB, Eisenman RN, Ness SA. 1998. Pim-1 kinase and p100 cooperate to enhance c-Myb activity. Mol Cell 2: 417-425.

Li CL, Yang WZ, Chen YP, Yuan HS. 2008. Structural and functional insights into human Tudor-SN, a key component linking RNA interference and editing. Nucleic Acids Res 36: 3579-3589.

Liu K, Chen C, Guo Y, Lam R, Bian C, Xu C, Zhao DY, Jin J, MacKenzie F, Pawson T, et al. 2010. Structural basis for recognition of arginine methylated Piwi proteins by the extended Tudor domain. Proc Natl Acad Sci 107: 18398-18403.

Morita Y, Shibutani T, Nakanishi N, Nishikura K, Iwai S, Kuraoka I. 2013 Human endonuclease $\mathrm{V}$ is a ribonuclease specific for inosine-containing RNA. Nat Commun 4: 2273.

Musiyenko A, Majumdar T, Andrews J, Adams B, Barik S. 2012. PRMT1 methylates the single Argonaute of Toxoplasma gondii and is important for the recruitment of Tudor nuclease for target RNA cleavage by antisense guide RNA. Cell Microbiol 14: 882-901.

Paukku K, Yang J, Silvennoinen O. 2007. Tudor and nuclease-like domains containing protein p100 function as coactivators for signal transducer and activator of transcription 5. Mol Endocrinol 17: 1805-1814.

Petoukhov MV, Franke D, Shkumatov AV, Tria G, Kikhney AG, Gajda M, Gorba C, Mertens HD, Konarev PV, Svergun DI. 2012. New developments in the ATSAS program package for small-angle scattering data analysis. J Appl Crystallogr 45: 342-350.

Receveur-Brechot V, Durand D. 2012. How random are intrinsically disordered proteins? A small angle scattering perspective. Curr Protein Pept Sci 13: 55-75.

Samuel CE. 2011. Adenosine deaminases acting on RNA (ADARs) are both antiviral and proviral. Virology 411: 180-193.

Scadden AD. 2005. The RISC subunit Tudor-SN binds to hyper-edited double-stranded RNA and promotes its cleavage. Nat Struct Mol Biol 12: 489-496.

Scadden ADJ, Smith CWJ. 2001. Specific cleavage of hyper-edited dsRNAs. EMBO J 20: 4243-4252.

Serra MJ, Smolter PE, Westhof E. 2004. Pronounced instability of tandem IU base pairs in RNA. Nucleic Acids Res 32: 1824-1828.

Shaw N, Zhao M, Cheng C, Xu H, Saarikettu J, Li Y, Da Y, Yao Z, Silvennoinen O, Yang J, et al. 2007. The multifunctional human p100 protein 'hooks' methylated ligands. Nat Struct Mol Biol 14: 779-784. 


\section{Li et al.}

Sundström JF, Vaculova A, Smertenko AP, Savenkov EI, Golovko A, Minina E, Tiwari BS, Rodriguez-Nieto S, Zamyatnin AA Jr, Välineva T, et al. 2009. Tudor staphylococcal nuclease is an evolutionarily conserved component of the programmed cell death degradome. Nat Cell Biol 11: 1347-1354.

Svergun DI. 1992. Determination of the regularization parameter in indirect-transform methods using perceptual criteria. J Appl Cryst 25: 495-503.

Tria G, Mertens HDT, Kachala M, Svergun DI. 2015. Advanced ensemble modelling of flexible macromolecules using X-ray solution scattering. IUCrJ 2: 207-217.

Tsuchiya $\mathrm{N}$, Ochiai $\mathrm{M}$, Nakashima $\mathrm{K}$, Ubagai $\mathrm{T}$, Sugimura $\mathrm{T}$, Nakagama H. 2007. SND1, a component of RNA-induced silencing complex, is up-regulated in human colon cancers and implicated in early stage colon carcinogenesis. Cancer Res 67: 9568-9576.

Välineva T, Yang J, Palovuori R, Silvennoinen O. 2005. The transcriptional co-activator protein p100 recruits histone acetyltransferase activity to STAT6 and mediates interaction between the CREB-binding protein and STAT6. J Biol Chem 280: 14989-14996.

Wan L, Lu X, Yuan S, Wei Y, Guo F, Shen M, Yuan M, Chakrabarti R, Hua Y, Smith HA, et al. 2014. MTDH-SND1 interaction is crucial for expansion and activity of tumor-initiating cells in diverse onco- gene- and carcinogen-induced mammary tumors. Cancer Cell 26: 92-105.

Wang C, Washida H, Crofts AJ, Hamada S, Katsube-Tanaka T, Kim D, Choi SB, Modi M, Singh S, Okita TW. 2008. The cytoplasmic-localized, cytoskeletal-associated RNA binding protein OsTudor-SN: evidence for an essential role in storage protein RNA transport and localization. Plant J 55: 443-454.

Watkins NE Jr, SantaLucia J Jr. 2005. Nearest-neighbor thermodynamics of deoxyinosine pairs in DNA duplexes. Nucleic Acids Res 33: 6258-6267.

Yang W, Chendrimada TP, Wang Q, Higuchi M, Seeburg PH, Shiekhattar R, Nishikura K. 2006. Modulation of microRNA processing and expression through RNA editing by ADAR deaminases. Nat Struct Mol Biol 13: 13-21.

Yang J, Valineva T, Hong J, Bu T, Yao Z, Jensen ON, Frilander MJ, Silvennoinen O. 2007. Transcriptional co-activator protein p100 interacts with snRNP proteins and facilitates the assembly of the spliceosome. Nucleic Acid Res 35: 4485-4494.

Yoo BK, Santhekadur PK, Gredler R, Chen D, Emdad L, Bhutia S, Pannell L, Fisher PB, Sarkar D. 2011. Increased RNA-induced silencing complex (RISC) activity contributes to hepatocellular carcinoma. Hepatology 53: 1538-1548. 

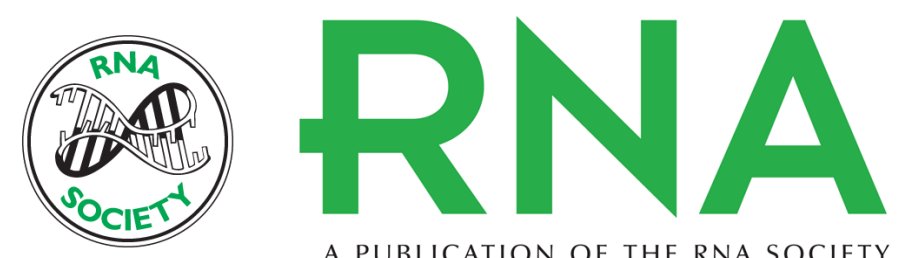

A PUBLICATION OF THE RNA SOCIETY

\title{
Tudor staphylococcal nuclease is a structure-specific ribonuclease that degrades RNA at unstructured regions during microRNA decay
}

\author{
Chia-Lung Li, Wei-Zen Yang, Zhonghao Shi, et al.
}

RNA 2018 24: 739-748 originally published online February 13, 2018

Access the most recent version at doi:10.1261/rna.064501.117

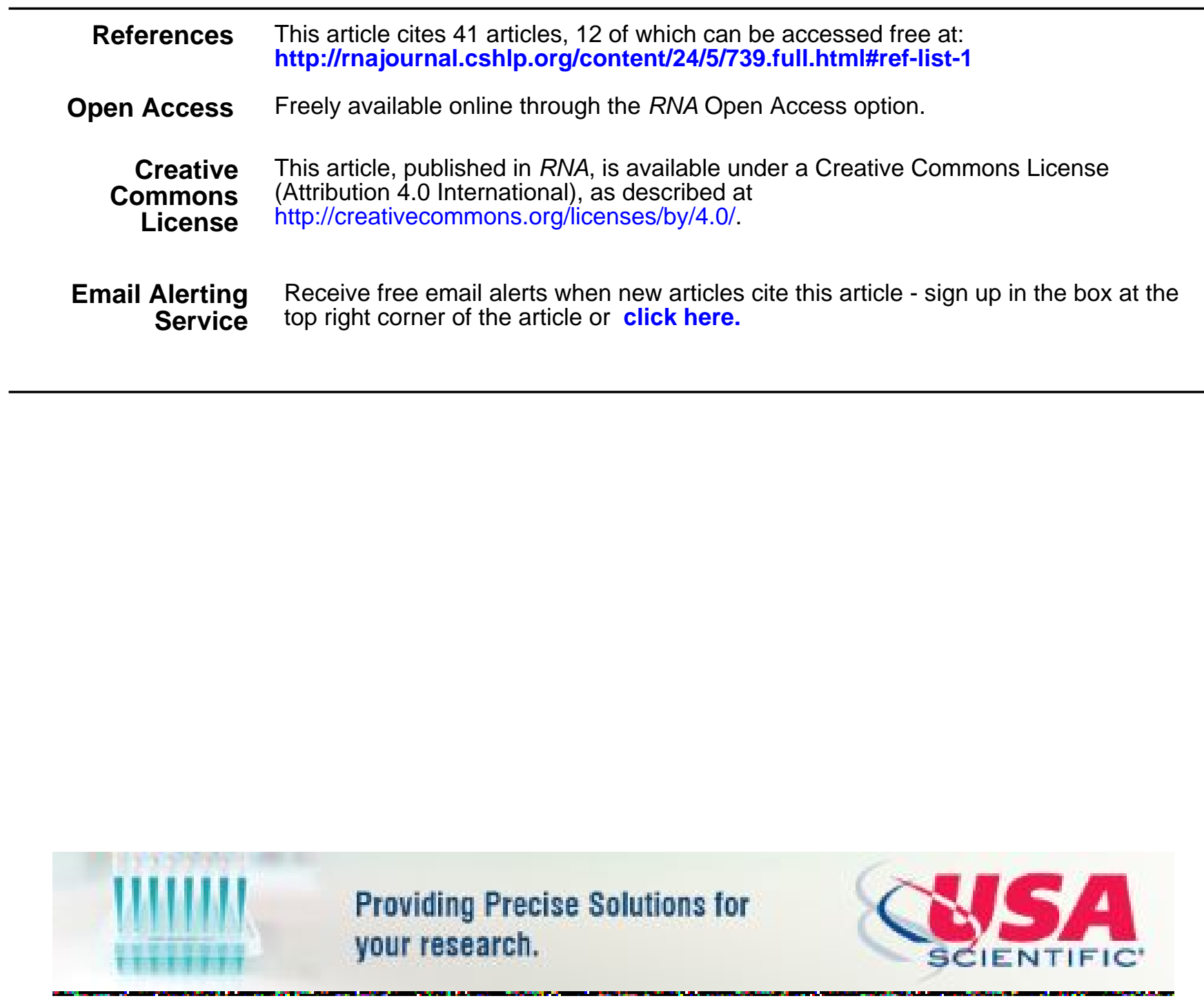

To subscribe to $R N A$ go to:

http://rnajournal.cshlp.org/subscriptions

(C) 2018 Li et al.; Published by Cold Spring Harbor Laboratory Press for the RNA Society 